\title{
Probing energy transfer in an ensemble of silicon nanocrystals
}

\author{
H. Jayatilleka, ${ }^{1}$ D. Diamare, ${ }^{1}$ M. Wojdak, ${ }^{1}$ A. J. Kenyon, ${ }^{1, a)}$ C. R. Mokry, ${ }^{2}$ P. J. Simpson, ${ }^{2}$ \\ A. P. Knights, ${ }^{3}$ I. Crowe, ${ }^{4}$ and M. P. Halsall ${ }^{4}$ \\ ${ }^{1}$ Department of Electronic and Electrical Engineering, University College London, Torrington Place, London \\ WCIE 7JE, United Kingdom \\ ${ }^{2}$ Department of Physics and Astronomy, The University of Western Ontario, 1151 Richmond Street, London, \\ Ontario N6A 3K7, Canada \\ ${ }^{3}$ Department of Engineering Physics, McMaster University, 1280 Main Street West, Hamilton, Ontario L8S \\ 4L7, Canada \\ ${ }^{4}$ Photon Sciences Institute and School of Electrical and Electronic Engineering, University of Manchester, \\ Manchester M13 9PL, United Kingdom
}

(Received 1 May 2011; accepted 28 June 2011; published online 9 August 2011)

\begin{abstract}
Time-resolved photoluminescence measurements of silicon nanocrystals formed by ion implantation of silicon into silicon dioxide reveal multi-exponential luminescence decays. Three discrete time components are apparent in the rise and decay data, which we associate with different classes of nanocrystals. The values of decay time are remarkably constant with emission energy, but the relative contributions of the three components vary strongly across the luminescence band. In keeping with the quantum confinement model for luminescence, we assign emission at high energies to small nanocrystals and that at low energies to large nanocrystals. By deconvolving the decay data over the full emission band, it is possible to study the migration of excitation from smaller (luminescence donor) to larger (luminescence acceptor) nanocrystals. We propose a model of diffusion of excitation between neighboring nanocrystals, with long lifetime emission being from the largest nanocrystal in the local neighborhood. Our data also allow us to study the saturation of acceptor nanocrystals, effectively switching off excitation transfer, and Auger recombination in non-interacting nanocrystals. (C) 2011 American Institute of Physics.
\end{abstract}

[doi:10.1063/1.3622151]

\section{INTRODUCTION}

Luminescence from nanostructured silicon (principally nanowires, nanoclusters, and nanocrystals) is of great technological interest because of its potential as a route to silicon-based optical sources for silicon photonics. Beginning with the observation by Canham in 1990 of bright emission from porous silicon, ${ }^{1}$ numerous groups have studied the optical properties of nanometre-scale silicon wires, clusters, and crystals. ${ }^{2}$ Despite reports of electroluminescence ${ }^{3-6}$ and even some evidence of optical gain from nanocrystals, ${ }^{7}$ the field remains controversial, with a number of open questions still persisting about the source of optical emission. Competing explanations include radiative recombination of confined excitons, ${ }^{8,9}$ luminescent surface states, ${ }^{10-12}$ combined emission from nanoclusters and nanocrystals, ${ }^{13}$ and defect emission. ${ }^{14,15}$

In the case of silicon nanoclusters and nanocrystals, the picture is further complicated by the presence of a distribution of nanocluster/nanocrystal sizes and inter-cluster separations. Samples containing nanoclusters generally consist of a silicon dioxide matrix containing a solid solution of nanoclusters, which, depending on the thermal history of the sample, may be amorphous or crystalline. Samples may be produced by ion implantation of silicon ions into $\mathrm{SiO}_{2},{ }^{16}$ by

\footnotetext{
a) Author to whom correspondence should be addressed. Electronic mail: t.kenyon@ee.ucl.ac.uk.
}

chemical vapor deposition (e.g., plasma-enhanced CVD), ${ }^{9}$ or by techniques such as sputtering or reactive evaporation. In all cases, a distribution of nanocluster sizes and separations is unavoidable. Transmission electron microscope images of such samples typically show a lognormal distribution of nanocluster sizes with mean diameters in the 1-4 $\mathrm{nm}$ range.

Many of the observed luminescence properties of ensembles of silicon nanocrystals can be explained by the quantum confinement model ${ }^{17}$ - particularly emission in the near-infra-red, though models for emission in the visible remain more controversial. In this model, carrier confinement in three dimensions within crystals smaller than the Bohr radius of the free exciton produces a widening of the electronic bandgap. Effective mass theory yields a fair approximation to the dependence of bandgap on nanocrystal radius $\left(E_{g} \propto 1 / r^{2}\right)$ for larger nanocrystals, though in many cases, the dependence on nanocrystal radius is somewhat weaker than $1 / \mathrm{r}^{2}$, thanks to the finite potential barrier at the nanocrystal surface. ${ }^{2}$ Although the approximation is reported to break down for smaller nanocrystals, in which surface states may dominate, ${ }^{18}$ inter-band excitonic recombination is a good model for photon emission in the near-ir and predicts that nanocrystals of different sizes will emit photons of different energies. It is also well-established that the excitation cross section of silicon nanocrystals varies strongly with crystal size, with larger nanocrystals exhibiting larger crosssections. ${ }^{19}$ Given a broad distribution of nanocrystal sizes, the resultant optical emission band will be broadened as a 
result of the corresponding range of bandgap energies. ${ }^{2}$ Recent work on single-dot spectroscopy has revealed a complex picture of competing non-radiative recombination processes, including phonon coupling, non-radiative surface states, such as the $\mathrm{P}_{\mathrm{b}}$ center, multi-exciton interactions, and Auger effects, and also emphasizes the role of size dispersion in producing the broad luminescence band that is characteristic of this material. ${ }^{20}$

Measurements of time-resolved photoluminescence from silicon nanocrystals reveal typically that decays are non-exponential. ${ }^{21-23}$ Multi-exponential and stretched-exponential models have been used to fit experimental data, and physical models to explain the observations include excitation migration, competing non-radiative decays, and disorder. $^{2}$ There have been suggestions that the stretched exponential decay is inherent in photoluminescence decays of crystalline material with trap states. ${ }^{24}$ Distributions of decay time constants, extracted from stretched exponential fits to experimental data, ${ }^{25}$ are also often explained in terms of size distributions of nanoclusters or nanocrystals, with the decay rate being in some way inversely proportional to the cluster size. Increasing decay times with emission wavelength are commonly reported. However, in contrast to other data in the literature, we find that luminescence decays of silicon nanocrystals are best described using a superposition of three discrete decay times, which vary little over the luminescence band, though the relative contribution of each band varies strongly with wavelength. Our data suggest the presence of three distinct classes of nanocrystal, and we explain our observations in terms of migration of excitation from small to large silicon nanocrystals. We propose that our measurements allow us to probe directly luminescence donor and acceptor populations.

\section{EXPERIMENTAL DETAILS}

A $430 \mathrm{~nm}$ thick thermal oxide layer was grown on a (100) $\mathrm{Si}$ wafer by wet oxidation at $1000{ }^{\circ} \mathrm{C}$. $\mathrm{Si}^{+}$ions with an energy of $90 \mathrm{keV}$ were implanted into the oxide layer to a total dose of $1.4 \times 10^{17} \mathrm{~cm}^{-2}$. Transport of ions in matter (TRIM) calculations showed that the implanted ions had a range of $127 \mathrm{~nm}$ into the oxide layer, with the distribution having a straggle of $42 \mathrm{~nm}$. Post-implantation, the sample was annealed in two separate stages: first at $1070{ }^{\circ} \mathrm{C}$ for $3 \mathrm{~h}$ in $\mathrm{N}_{2}$ and then at $450{ }^{\circ} \mathrm{C}$ for $1 \mathrm{~h}$ in a mixture of $95 \% \mathrm{~N}_{2}$ and $5 \% \mathrm{H}_{2}$.

Note that the detailed photoluminescence study was performed on one specific sample, though we have measured multiple exponential decays from a range of silicon nanocrystal- and nanoclusters-containing samples prepared using ion implantation or plasma enhanced chemical vapor deposition (PECVD). Non-exponential decays are a commonly observed feature of such materials.

Transmission electron microscope (TEM) images of nanocrystals were taken with a JEOL 2010 field emission TEM/STEM with a Gatan electron energy loss spectrometer energy filter. The TEM images were taken from a sample prepared under the same conditions as those described in the previous paragraph. Further details of the structural charac- teristics of samples prepared in this way may be found elsewhere. $^{26}$

Photoluminescence (PL) was excited by a diode pumped solid state (DPSS) laser emitting at $473 \mathrm{~nm}$, producing 40 $\mathrm{mW}$ power at the sample, focused on an area of $5.4 \times 10^{-4}$ $\mathrm{cm}^{2}$. Modulation of the laser beam was achieved using a Pockels cell at a frequency of $548 \mathrm{~Hz}$. The rise and fall time of the laser intensity was limited by the response of the Pockels cell to approximately $15 \mathrm{~ns}$. The luminescence signal was analyzed by a Bentham M300 single grating monochromator with a grating of 1200 lines/mm and was detected by a Hamamatsu infra-red sensitive photomultiplier tube (PMT), model number R5509-72. In one set of experiments, the signal from the PMT was amplified using a current preamplifier with a gain of $10 \mu \mathrm{A} / \mathrm{V}$, and waveforms were recorded with a digital oscilloscope. In a separate set of photon counting experiments, the PMT signal was amplified by a Becker \& Hickl ACA-4 $1.8 \mathrm{GHz}$ wide-band amplifier giving $35 \mathrm{~dB}$ gain and detected using a Becker \& Hickl MSA300 multiscalar analyzer, which has a time resolution of 5 ns. Photoluminescence rise and decay data were obtained at 46 detection wavelengths from $700 \mathrm{~nm}-1060 \mathrm{~nm}$. All measurements were performed at room temperature. Data were fitted using a variety of decay functions (multiple exponential and stretched exponential) using Matlab, and luminescence rise and fall times extracted. In separate experiments, the photon flux dependence of both the photoluminescence line shape and the relative intensities and time constants of the three decay components were measured using the same experimental set-up.

\section{RESULTS}

Fig. 1 shows a TEM image of a sample prepared under similar conditions to the sample discussed here. Silicon nanocrystals are clearly visible as the dark circles against the background speckle from the oxide matrix.

Figure 2 shows the dependence of the time-integrated photoluminescence spectrum on incident photon flux. A

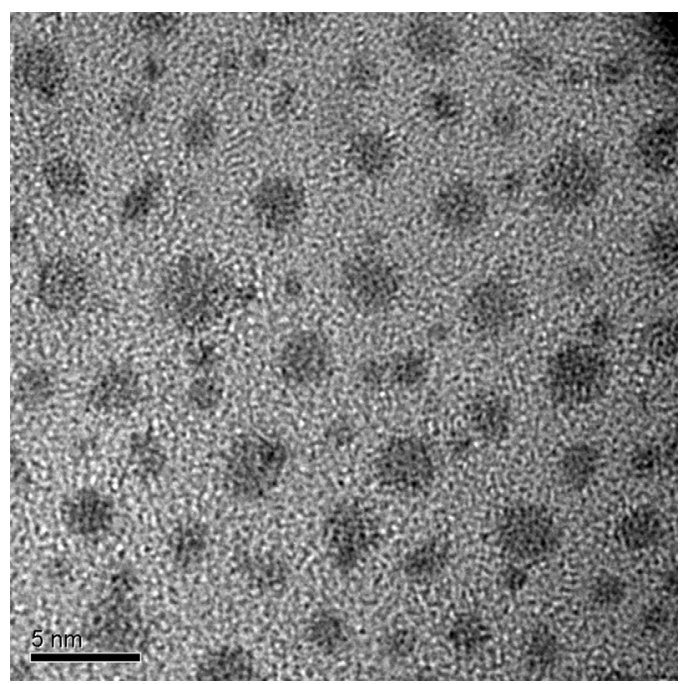

FIG. 1. TEM image of a sample prepared under the same conditions as the sample studied in this work, showing silicon nanoclusters. 

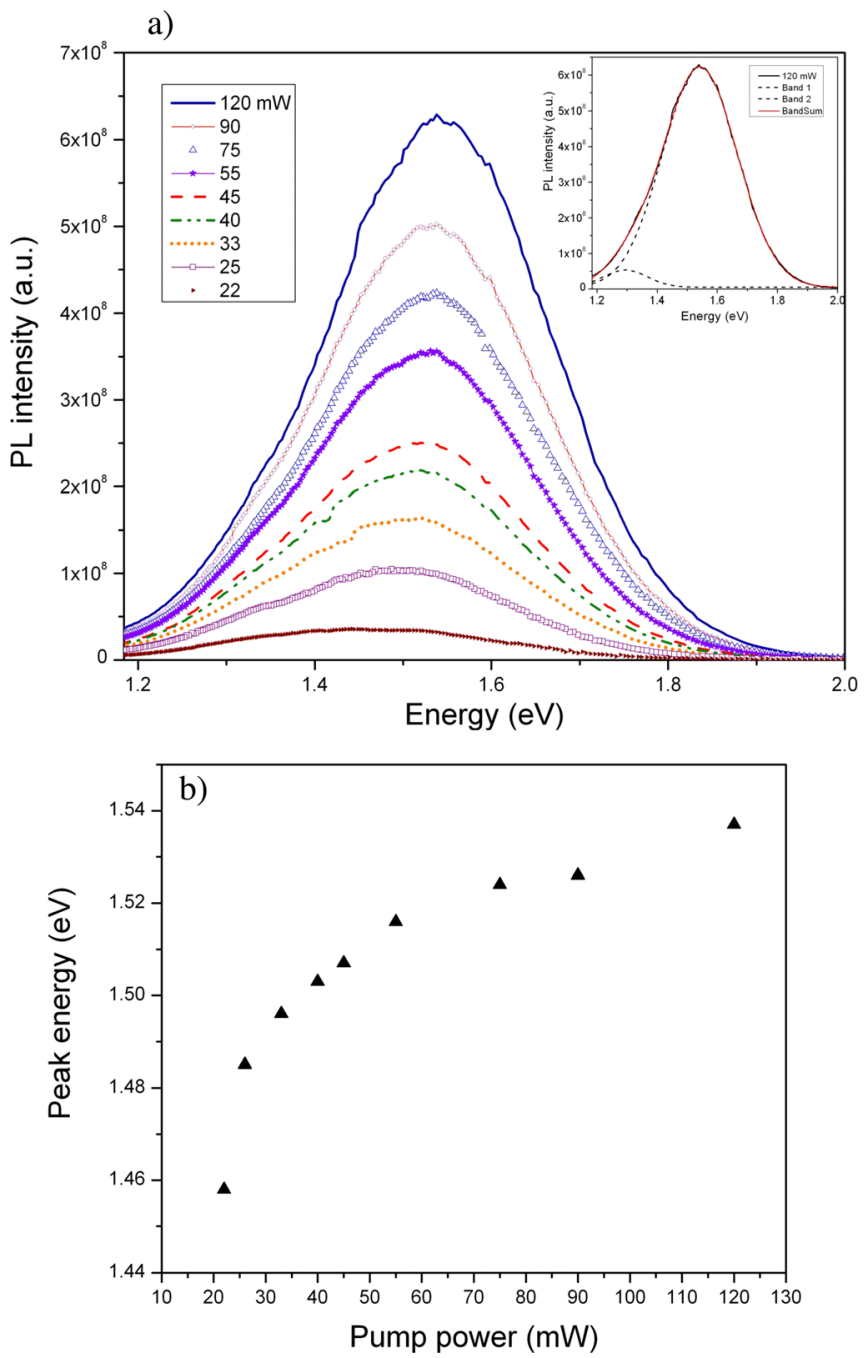

FIG. 2. (Color online) a) PL spectra as a function of pump laser power pumped at $473 \mathrm{~nm}$. a) Inset: Deconvolution of spectrum taken using a pump power of $340 \mathrm{~mW}$, showing two constituent emission bands. Spectra corrected for the system response. b) Peak position as a function of pump power.

broad peak, centered around $1.5 \mathrm{eV}(830 \mathrm{~nm})$, is typically reported as characteristic of emission from silicon nanocrystals. The primary luminescence mechanism in this wavelength range is assumed to be radiative recombination of confined excitons within nanocrystals, and the peak broadening is attributed to the broad nanocrystal size distribution. Clearly observable is a blue-shift in the peak energy with increasing photon flux (shown in more detail in the lower graph), but more significantly, we find that the spectra are asymmetric, and each can be deconvolved into two separate bands: one centered around 1.3-1.4 eV and the larger of the two around 1.5-1.55 eV. We assume that emission at high energies (short wavelengths) is from small nanocrystals (i.e., those with large bandgap energies) and that at low energies is from large nanocrystals. The peak shape is, therefore, to a first approximation, a convolution of bandgap modification by quantum confinement effects and the nanocrystal size distribution.

Figure 3 shows representative PL rise and decay curves, measured at a detection wavelength of $800 \mathrm{~nm}(1.55 \mathrm{eV})$.
The photon counting set-up was used for this experiment, but very similar results were obtained using the digital oscilloscope - the differences between results from the two set-ups were negligible. The curves are clearly not single exponentials, and the fitted curves drawn on the data illustrate the quality of fit obtained using a triple exponential fitting function. A number of different fitting functions were tested in addition to the triple exponential - double, quadruple, and stretched exponential functions - but all of the rise and decay curves obtained could be best fitted by three exponentials, in each case yielding acceptably low $\chi^{2}$ values $\left(1.71 \times 10^{-2}\right.$ and $3.90 \times 10^{-2}$ for the curves shown in Fig. 3, for example). As a further test, we utilized a numerical approach to obtaining the distribution of time constants in decay data, mirroring that adopted by Delerue et al. ${ }^{27}$ and in our previous work on time resolved luminescence from silicon nanocluster-sensitized erbium. ${ }^{28}$ We treated the observed PL decay as a discrete sum of exponential terms weighted by a distribution of time constants

$$
I(t)=\sum_{1}^{i} \frac{1}{\tau_{i}} A_{i} \exp \left(-\frac{t}{\tau_{i}}\right) .
$$

Determining the distribution of the constants $A_{i}$ over $i$ allows us to obtain an approximation to the distribution of decay constants. We performed such an analysis of our timeresolved PL data. We chose 80 values of $\tau$, ranging between 1 and $720 \mu \mathrm{s}$, and fitted test experimental decay curves using a Levenberg-Marquardt fitting algorithm. We initially confirmed the accuracy of this method by performing fits on analytically generated known distributions of single and multiple exponential data. We found that this method converged to a distribution centered on three decay times, further confirming our selection of a triple exponential function. Of course, this result should be taken only as indicative, as the fixed values of decay time used in this method preclude an accurate determination of the real values of the three components. As a result, the chi squared value for this function after converging on three lifetimes is slightly higher than that for a three exponential fit in which the lifetime values are allowed to vary. Table I shows the chi squared values obtained from fitting the $800 \mathrm{~nm}$ decay curve with different functions.

To further emphasize the superior quality of the triple exponential fit over that of the stretched exponential, Fig. 4 shows a plot of residual values for both triple and stretched exponential fits for a decay curve taken at $800 \mathrm{~nm}$. Data were normalized to a peak value of 1 , and residuals are shown for the first $250 \mu \mathrm{s}$, demonstrating that the triple exponential fit is the better of the two. In the case of the quadruple exponential fit, two of the components converged to the same decay time, resulting in a triple exponential.

Figure 5 illustrates the variation in the time constants of the three lifetime components with photon energy. There is surprisingly little variation in the individual components across the PL band, with the longest being around $225 \mu \mathrm{s}$, the medium around $75 \mu \mathrm{s}$, and the shortest around $20 \mu \mathrm{s}$. There is a tendency at lower energies for the longest component to shorten with increasing photon energy, which is in keeping with observations in the literature, ${ }^{29}$ but the 

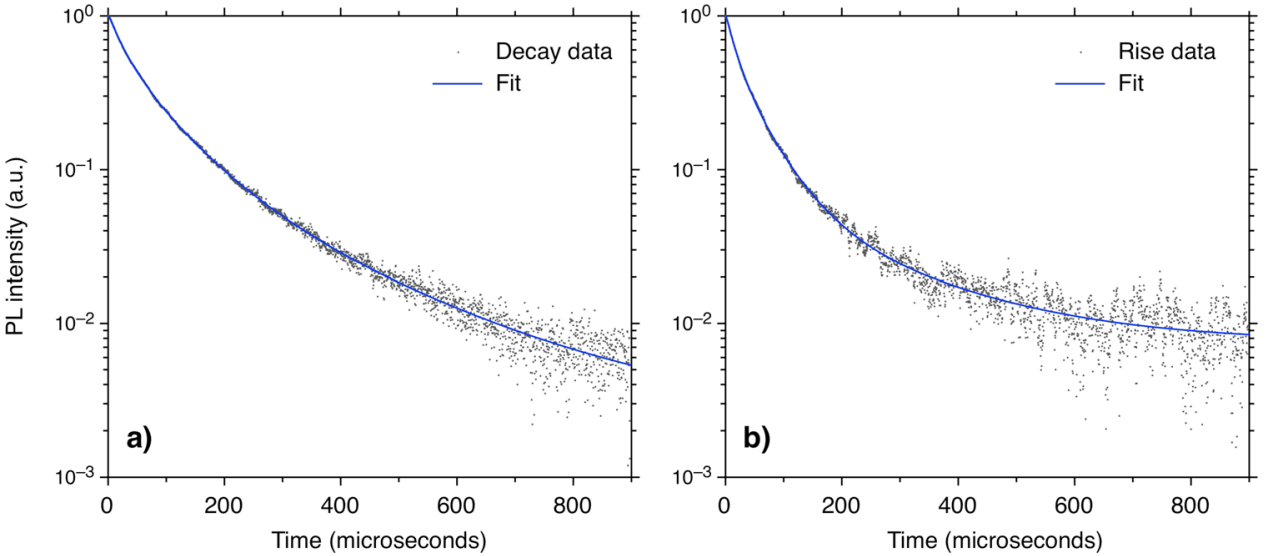

FIG. 3. (Color online) PL transients recorded using a photon counting set-up. (a) Decay; (b) rise (inverted for comparison with decay data). Solid lines are triple exponential fits. uncertainties in fitting data at the extremes of the luminescence band, where the signal is lowest, make it difficult to draw any firm conclusions. Even if it is the case that the lifetime of this component varies with photon energy and, hence, by implication with nanocrystal size, the observation that there are three distinct lifetime components in the spectrum of our sample remains valid. Figure 6 illustrates the relative contribution to the total photoluminescence signal of the three separate decay time components. To construct this figure, triple exponential fits were performed to decay curves obtained at each of the 46 detection wavelengths and the weights $\left(A_{i}\right.$ values $)$ of each of the three components plotted as a function of photon energy. Because of the variation in signal intensity across the luminescence band, fitting accuracy varied from point to point, but an indication of the quality of fit is given by the spread in $\tau$ values evident in Fig. 5 . A greater spread is evident at the extremes of the luminescence band, while, at the center, the lifetime values are much more constant. It is clear from Fig. 6 that the high photon energies are dominated by the short- and medium-time components, while, at low photon energies, the long decay component becomes apparent.

Figure 7 details the dependence of the three lifetime components on photon flux. The shortest of the three shows a small, but significant, increase with photon flux, while the medium component shows a slight reduction, and data from the longest component is rather inconclusive.

\section{DISCUSSION}

The three time constants in photoluminescence decay data show surprisingly little variation across the photoluminescence band, notwithstanding the possible weak dependence of the long component on photon energy; instead, the most significant trend is that of the changing relative contributions of the short-, medium-, and long-components at different photon energies. This is perhaps surprising, as it is often reported that luminescence decay times increase with nanocrystal size and decreasing photon energy. ${ }^{29}$ Nevertheless, the quality of the triple exponential fits to the data suggests the presence of three distinct populations of emitting nanocrystals, with any link between decay time and nanocrystal size being a secondary effect. Assuming a dependence of PL emission energy on nanocrystal size in line with quantum confinement effects, we surmise that short or medium PL lifetimes $(\sim 20 \mu$ s or $\sim 75 \mu \mathrm{s})$ are seen predominantly in small nanocrystals, with minor contributions from large crystals, while the long lifetime component $(\sim 225 \mu \mathrm{s})$ is only seen for larger nanocrystals. We note further that the spectral distributions of the short and medium lifetime components are strikingly similar, with peaks around $1.54 \mathrm{eV}$, while that of the long component peaks at a lower energy ( $\sim 1.40 \mathrm{eV}$ ), corresponding to an apparent shoulder in the short and medium lifetime spectra. This is strikingly similar to the deconvolution of the time-integrated total PL spectra into two bands. On the basis of these observations, we conclude that the same size distribution of nanocrystals underlies the short and medium lifetime components.

In order to explain these data - beginning with the $\sim 1.40 \mathrm{eV}$ band that appears in all three spectra - we note that the proximity of silicon nanocrystals in this sample allows excitation migration between neighboring nanocrystals. Typical nanocrystal separations in this material are of the order of 5-7 nm, with many appearing to be far less (see Fig. 1). This is an important observation, as Allan and Delerue predict that energy transfer due to multipolar interactions between silicon nanocrystals can only occur for very small inter-crystal separations. ${ }^{30}$ A particular feature of excitation

TABLE I. Chi squared results for different fits to the normalised $800 \mathrm{~nm}$ decay data.

\begin{tabular}{lcr}
\hline \hline Fitting function & Functional form & $\chi^{2}$ \\
\hline Stretched exponential & $I=I_{0} e^{-\left(\frac{t}{t^{\beta}}\right)^{\beta}}$ & $6.175 \times 10^{-2}$ \\
Triple exponential & $I=A_{1} e^{-\left(\frac{t}{\tau_{1}}\right)}+A_{2} e^{-\left(\frac{t}{\tau_{2}}\right)}+A_{3} e^{-\left(\frac{t}{\tau_{3}}\right)}$ & $1.709 \times 10^{-2}$ \\
Discrete exponentials (80 different fixed values of tau) & $I=A_{1} e^{-\left(\frac{t}{\tau_{1}}\right)}+A_{2} e^{-\left(\frac{t}{\tau_{2}}\right)}+\ldots A_{80} e^{-\left(\frac{t}{\tau_{80}}\right)}$ & $1.711 \times 10^{-2}$ \\
\hline \hline
\end{tabular}




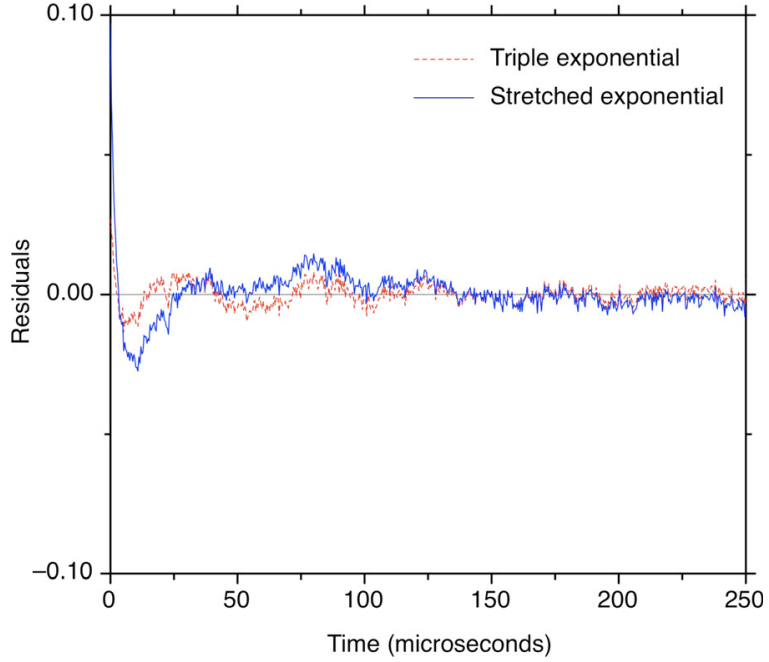

FIG. 4. (Color online) Fitting residuals for triple and stretched exponential fits to decay data taken at $800 \mathrm{~nm}(1.55 \mathrm{eV})$. Blue (solid line): stretched exponential; red (dotted line): triple exponential.

exchange is that it will occur preferentially from small to large nanocrystals and not vice-versa, as a consequence of the larger bandgap energy of the smaller nanocrystals. The recombination of excitons across the narrow bandgap of large nanocrystals will not provide sufficient energy to excite carriers in nearby wide bandgap small nanocrystals. We define the nanocrystals providing excitation as luminescence "donors" and those receiving it as luminescence "acceptors". If the concentration of nanocrystals is sufficiently high, sequential migration of excitation from donors to acceptors is possible, with emission ultimately occurring from the largest nanocrystal in the local environment. Of course, this population can be excited either directly by pump photons or indirectly by transfer of excitation from nearby crystals; hence, there are two parallel excitation routes for this class of nanocrystals, but in either case it is not possible for these nanocrystals to transfer their excitation to nearby nanocrystals. We would therefore expect the nanocrystals that are the larg-

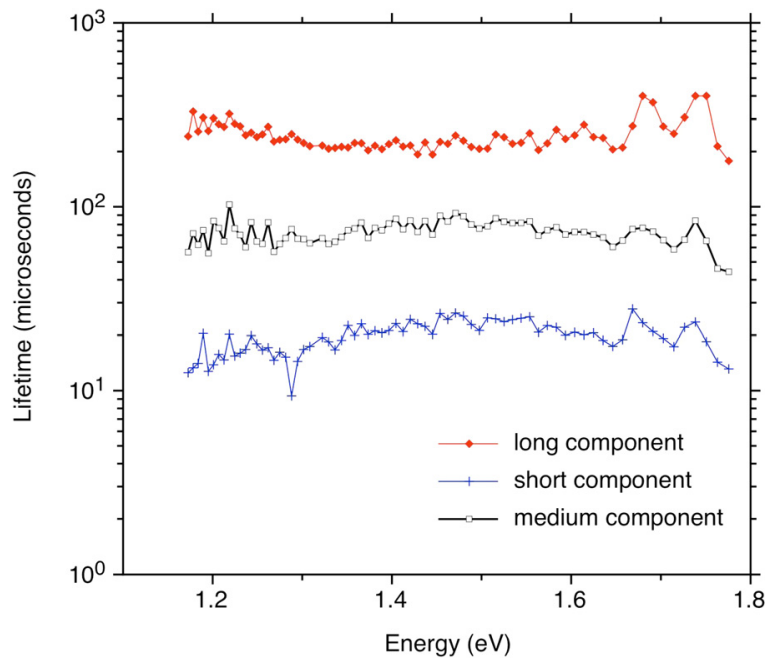

FIG. 5. (Color online) Variation of the time constants of the three components of PL decay data with photon energy extracted from fits to PL data.

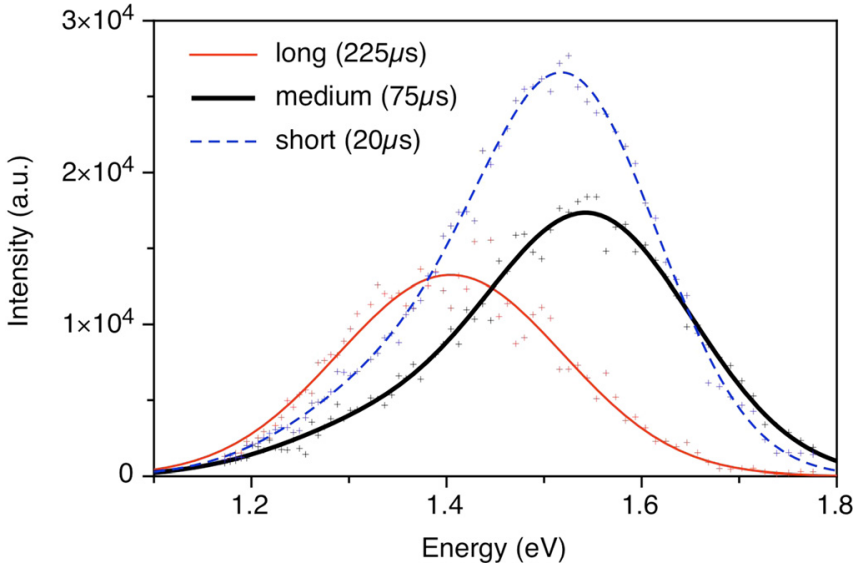

FIG. 6. (Color online) Varying contributions of the three decay components to the PL signal as a function of detection energy. Red (light solid line) long component; black (heavy solid line) medium component; blue (dashed line) short component. Lines are guides for the eye.

est in their neighborhood to have longer luminescence lifetimes than other, smaller, donor nanocrystals that experience the transfer of excitation as a non-radiative decay. We note further that this diffusion of excitation from smaller nanocrystals may result in emission from the largest nanocrystals in the neighborhood being dominated by that from indirectly excited "acceptor" nanocrystals, depending on the relative populations of large and small nanocrystals.

The probability $(P(r))$ of a given nanocrystal of radius $r$ being the largest in its neighborhood is given by the integral of the size distribution of nanocrystals, $\zeta(r)$ :

$$
P(r)=\int_{0}^{r} \zeta(r) d r
$$

Hence, the size distribution of nanocrystals that are the largest in their neighborhood is given by the product of this integral and the original size distribution

$$
\rho(r)=P(r) \zeta(r)=\zeta(r) \int_{0}^{r} \zeta(r) d r .
$$

In terms of the PL intensity $(I)$ as a function of energy ( $\propto 1 /$ $d^{2}$ ) from these nanocrystals, we have

$$
I(E) \propto \zeta(E)\left(1-\int_{0}^{E} \zeta(E) d E\right) .
$$

We are now in a position to perform a numerical integration of the luminescence data to test the validity of this hypothesis. To do this, we numerically integrate the total luminescence intensity from the lowest $\left(E_{0}\right)$ to the highest $\left(E_{n}\right)$ energy and calculate the value of $I(E)_{i}$ at each energy as a fraction of the total integral

$$
\int_{0}^{n} \zeta(E) d E=\frac{\sum_{i=0}^{i} I(E)_{i}}{\sum_{i=0}^{i=n} I(E)_{i}} .
$$

The element-by-element multiplication of $\int \zeta(E)_{i}$ and $I(E)_{i}$ gives the expected luminescence distribution (in energy) 

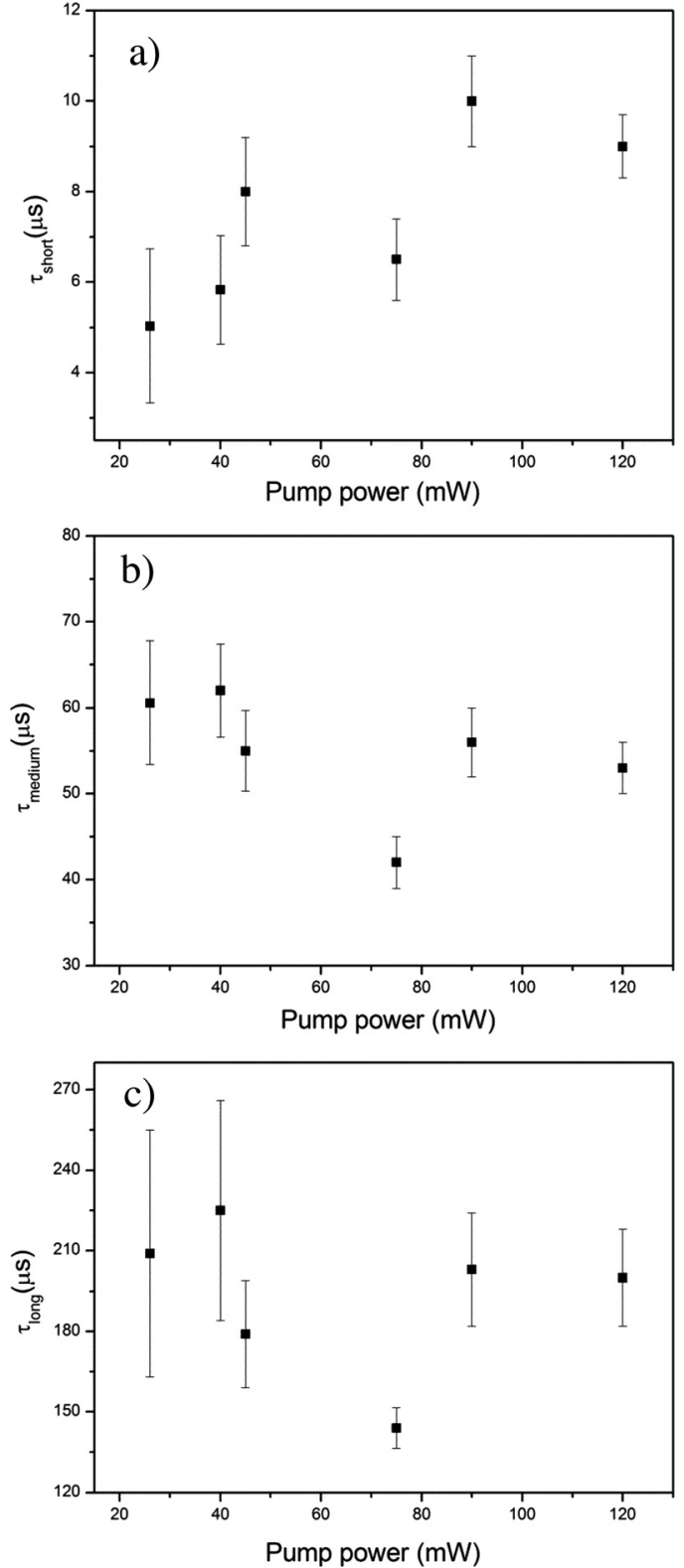

FIG. 7. Variation in the three lifetime components with pump power measured at an emission wavelength of $800 \mathrm{~nm}$. Pump wavelength $=473 \mathrm{~nm}$. a) Short component; b) medium component; c) long component.

$$
I(E)=\left(\frac{\sum_{i=0}^{i} I(E)_{i}}{\sum_{i=0}^{i=n} I(E)_{i}}\right) \times I(E)_{i} .
$$

As a first approximation, we thus expect to obtain the spectral shape of the long lifetime luminescence band by numerical integration of the total luminescence spectrum. Figure 8 shows the result of this calculation, with the measured long lifetime band superimposed. There is a reasonably good match between the calculated and measured curves - particularly given the uncertainties in both sets of data. Note that, in this calculation, we have made the assumption that the excitation cross-section of the nanocrystals does not vary with size. A more accurate model would allow for the very real variation in $\sigma$ with nanocrystal diameter, which would red-

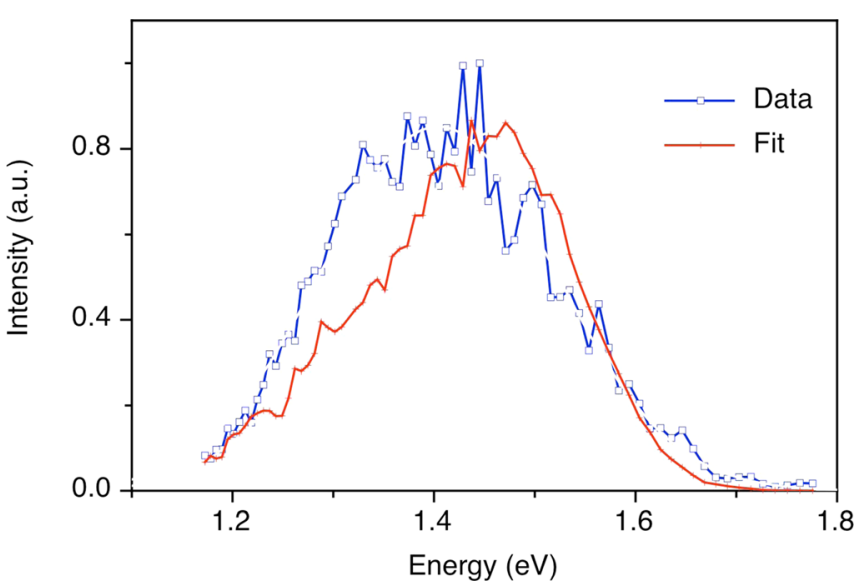

FIG. 8. (Color online) Comparison of the PL spectrum's long lifetime component (data) with the transformation of the total PL spectrum, using Eq. (6) to predict the shape of the PL spectrum of luminescent acceptors (fit). Data have been normalized to aid comparison.

shift the calculated spectrum of the large nanocrystals, bringing it more in line with the experimental data. Nevertheless, for our purposes, the results shown in Fig. 8 are indicative.

The luminescence lifetimes of donor nanocrystals will necessarily be shorter than those of the acceptors, the difference being due to the extra non-radiative decay process of excitation exchange. The presence of three lifetime components implies a further non-radiative decay channel - we discuss the implication of this below. We can therefore make an estimate of the relative quantum efficiency of the three bands by taking that of the long lifetime band as a baseline. In doing this, we are, of course, not taking the decay time of the long lifetime band to be purely radiative - instead, we are assuming that the lifetimes of the other two bands would be as long, but are instead shortened by various extra nonradiative processes, of which excitation transfer will be one. In this case, the relative quantum efficiencies of the mediumand short-decay time bands are given by the ratios of their lifetimes to that of the long lifetime band. Knowing these, the relative populations of the three types of nanocrystal can be obtained by dividing the luminescence intensity by the relative quantum efficiency. If all nanocrystals have the same inherent radiative lifetime, those suffering from more non-radiative decay (i.e., those with a low quantum efficiency) will make a smaller contribution to the photoluminescence signal than the size of their population would suggest. Thus, given the photoluminescence intensities from each class of nanocrystal, relative populations may be extracted by normalizing with respect to relative quantum efficiencies. The results, shown in Table II, clearly demonstrate that only a small percentage $(3.5 \%)$ of nanocrystals contribute to the longest decay time of $225 \mu$ s. Figure 9 further emphasizes this point by showing the photoluminescence spectra of the three decay components corrected for their relative quantum efficiencies. This observation is not surprising, as in order for a nanocrystal to have the longest PL lifetime, it must be both isolated from defects and other non-radiative recombination processes and be the largest nanocrystal in the local neighborhood (so no excitation migration can take place). It can, of course, be excited either 
TABLE II. Comparison of the three decay components' relative quantum efficiencies and populations. Data measured at $800 \mathrm{~nm}$ with a pump power of 40 $\mathrm{mW}$.

\begin{tabular}{lccccc}
\hline \hline Decay component & PL decay time $(\mu \mathrm{s})$ & Decay rate $\left(\mathrm{s}^{-1}\right)$ & Relative PL intensity (a.u.) & Relative quantum efficiency & Relative population \\
\hline Long & 225 & $4.4 \times 10^{3}$ & 1.0 & 1.0 & 1.0 \\
Medium & 75 & $1.3 \times 10^{4}$ & 1.34 & 0.333 & 4.02 \\
Short & 20 & $5.0 \times 10^{4}$ & 2.11 & 0.089 & 23.71 \\
\hline \hline
\end{tabular}

by direct photon absorption or by transfer of excitation from a nearby smaller nanocrystal, but in any case, only a small number of large nanocrystals will have the longest lifetime, a prediction that is borne out by our results.

We have noted already the striking similarity between the photoluminescence spectra of the fast and medium decay components, and note further that both can be decomposed into two Gaussian peaks: one at $1.37 \pm 0.05 \mathrm{eV}$ and a second at $1.54 \pm 0.02 \mathrm{eV}$, as indeed can the time integrated PL. Figure 10 illustrates this decomposition for the short lifetime component. This is strongly suggestive of a common origin for both bands, but two competing non-radiative quenching mechanisms producing two different luminescence lifetimes. We note further that the spectrum of the long lifetime band is centered at $1.40 \pm 0.01 \mathrm{eV}$, corresponding closely to the peak of the lower energy band evident in the other two spectra. This is again strongly indicative that the three low energy components share a common origin. Taken together with the results of the numerical integration of the luminescence bands (Eq. (6)), which generate a spectrum similar to the long lifetime band (Fig. 8), these observations suggest that, in each case, the low energy peak around $1.4 \mathrm{eV}$ originates from nanocrystals that have been excited by energy transfer from smaller nanocrystals. The time-integrated PL results can be explained by realizing that the largest nanocrystals have two parallel excitation pathways and will, therefore, contribute a disproportionately large fraction of the total photoluminescence signal, resulting in the appearance of a low-energy shoulder to the PL peak. The blueshift in the time-integrated PL peak energy with photon flux can be

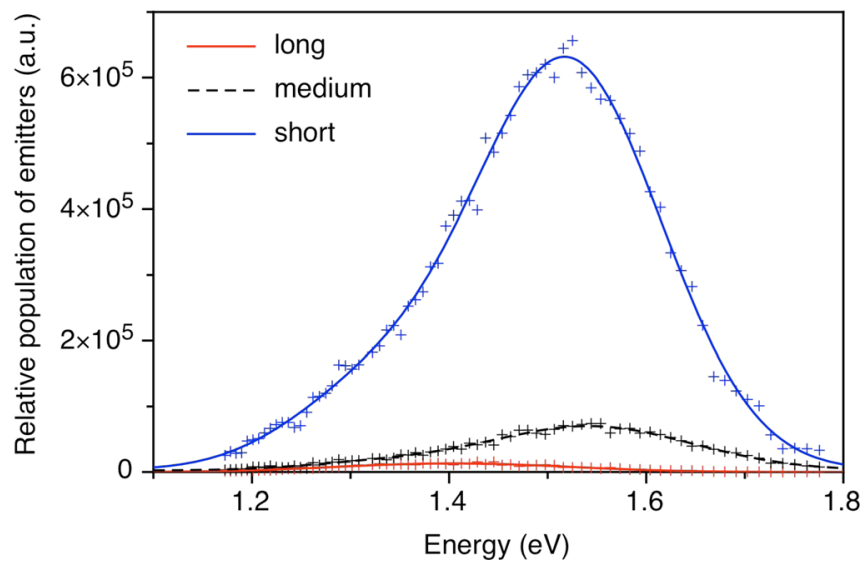

FIG. 9. (Color online) PL data for the three PL decay components corrected for their relative quantum efficiencies. Red (light solid line) long component; black (dashed line) medium component; blue (heavy solid line) short component. This gives an indication of the relative populations of nanocrystals contributing to each band. Clearly, the majority of nanocrystals have a short PL decay time, and only a small minority are responsible for the long component. explained by the variation in nanocrystal excitation cross-section with size. Large nanocrystals have a larger cross section than small ones and will, hence, saturate at lower photon fluxes. As the incident photon flux is increased, the net result is a progressive saturation of the PL from larger nanoclusters, yielding a blueshift in the PL peak energy with photon flux.

In order to fully explain our results, we propose the existence of three classes of nanocrystals:

1. Nanocrystals that cannot transfer excitation, either because they are isolated, or because they are the largest in the neighborhood, but nevertheless suffer from nonradiative recombination. This may be due to defects, surface states, or Auger effects - the latter of which will scale with photon flux, with consequences for the pump flux dependence of this lifetime component (see next paragraph). This class of nanocrystals would give rise to the medium lifetime component with a recombination rate of around $1.3 \times 10^{4} \mathrm{~s}^{-1}$. The largest nanocrystals in this class may be excited either directly or by transfer from other, smaller, interacting nanocrystals in the vicinity hence, the observation of a component centered around $1.4 \mathrm{eV}$ in the spectrum of this population.

2. Nanocrystals that interact with other, larger, nanocrystals in the vicinity. In this case, excitation transfer serves as a second non-radiative process with a higher rate than the non-radiative processes present in the first class of nanocrystals. The observed recombination rate of $5 \times 10^{4} \mathrm{~s}^{-1}$ gives rise to the short lifetime component in the photoluminescence. Again, we see a component peak centered around $1.4 \mathrm{eV}$ from nanocrystals that have been excited by transfer from smaller crystals, but are not the largest in

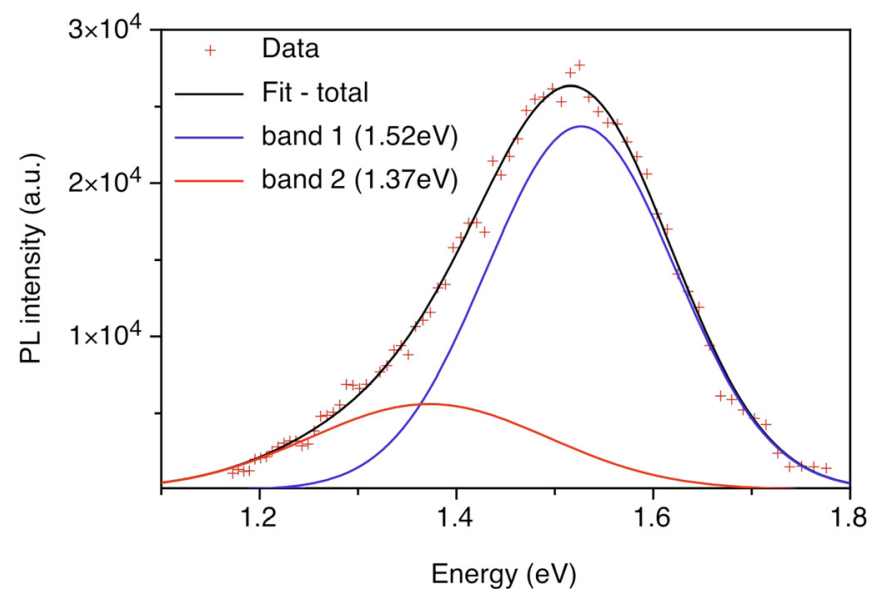

FIG. 10. (Color online) Fitting the short lifetime component PL data with two Gaussian peaks. Red (LH Gaussian peak) $1.37 \mathrm{eV}$ band; blue (RH Gaussian peak) $1.52 \mathrm{eV}$ band; black (bold solid line following data points) sum of two individual bands. 
the local environment and are therefore able to interact with nearby large nanocrystals. Significantly, as the number of large nanocrystals that are available to accept excitation from the smaller crystals is limited and, moreover, the excitation cross-sections of such crystals are larger than those of the smaller donor nanocrystals, it is possible to saturate the transfer process at higher photon fluxes. In such a case, we would expect to see an increase in the PL lifetime of the donor crystals at high photon fluxes. Figure 7 shows such an increase in the short lifetime component. The slight decrease in the decay time of the medium lifetime component with increasing photon flux is consistent with the origin of this component being non-interacting nanocrystals. Saturation of the acceptor nanocrystals has no effect on this population, and the decrease in PL lifetime is instead indicative of Auger deexcitation, which increases with photon flux, leading to a shorter PL lifetime.

3. Nanocrystals that are the largest in the neighborhood, and do not, therefore, transfer excitation to other nanocrystals and also do not suffer from the non-radiative processes that plague other isolated nanocrystals. Such nanocrystals will be free from surface defects and will be in a defectfree environment. These nanocrystals will give rise to the long lifetime component with a recombination rate of $4.4 \times 10^{3} \mathrm{~s}^{-1}$. Because these nanocrystals are the largest in their neighborhood, their spectrum is given by Eq. (6) and, in the case of our results, is approximated by a single Gaussian peak at $1.4 \mathrm{eV}$. The population of such nanocrystals will necessarily be small, in agreement with the observation that only $3.6 \%$ of the nanocrystals in our sample fall into this category.

Direct evidence for the existence of large nanocrystals that receive excitation through the two parallel paths of direct absorption and excitation transfer comes also from deconvolution of the time integrated photoluminescence spectrum, which also yields a low energy peak from those nanocrystals that are the largest in their local environment.

We emphasize that we are proposing three distinct classes of nanocrystals whose photoluminescence behavior is a consequence of both their relative size compared to neighboring crystals and to the local concentration of luminescence-quenching defects. We are not proposing that there are three distinct sizes of nanocrystals in our sample - in fact, TEM data can best be fitted with a single lognormal distribution (not shown). This is an important distinction.

Although it is not possible, on the basis of our results, to definitively exclude the possibility that the three lifetime components may be inherent to individual nanocrystals and may arise from some peculiarity of the radiative recombination process in silicon nanocrystals, we consider this unlikely. If different components were due to defects, impurities, or surface states, there is no reason to expect the emission spectra to match so closely. In fact, defect emission from $\mathrm{SiO}_{2}$ samples containing silicon nanocrystals is often reported, but at higher photon energies than those reported here. It is the striking similarities between the emission spec- tra of the different lifetime components that suggest different local environments for otherwise identical ensembles of nanocrystals. The simplest explanation that is consistent with our observations is that of different non-radiative recombination processes operating on otherwise similar populations of nanocrystals, giving rise to the different and discrete time constants in the luminescence decay data.

Furthermore, although our decay data can clearly be best fitted using three distinct time constants, we do not discount variations in the radiative rate of nanocrystals with nanocrystal size, as reported previously in the literature. It would be surprising if the radiative rates of nanocrystals of all sizes were the same, and our data shows some evidence of some variation with nanocrystal size. Nevertheless, this is a secondary effect to the appearance of three lifetime components in our time-resolved data. We propose, therefore, that these detailed time-resolved measurements discriminate between different classes of nanocrystal and allow us to probe directly luminescence donor and acceptor populations.

It would be informative to study the dependence of the complex decay dynamics we describe here on sample preparation and post-process annealing. Of particular interest would be the dependence of decay dynamics on nanocrystals size distributions. Such studies are underway.

\section{CONCLUSIONS}

Luminescence decay data from silicon nanocrystals produced by ion implantation have been studied in detail across the luminescence band attributed to radiative recombination of confined excitons. At all photon energies, the data can be best fitted by three discrete exponential decays. The fitting is robust and suggests the presence of distinct, but interacting, populations of nanocrystals. In the absence of discrete sizes in the nanocrystal size distribution, we propose that the data can be explained by a model that takes into account migration of excitation from small nanocrystals to neighboring large ones, with the longest lifetime emission tending to come from the largest nanocrystal in the neighborhood. This interpretation is supported by the observation that the shortest lifetime component, arising from luminescence donors, lengthens with increasing photon flux, suggesting saturation of the larger acceptor clusters, which have larger excitation cross-sections. Further evidence for the existence of discrete luminescence donor and acceptor nanocrystals comes from time-integrated photoluminescence results, indicating a population of large nanocrystals that are subject to both direct and indirect excitation. We are thus able to directly and separately probe the luminescence donor and acceptor nanocrystal populations.

\section{ACKNOWLEDGMENTS}

This work was supported by the UK Engineering and Physical Sciences Research Council. H.J. gratefully acknowledges the award of an Undergraduate Research Bursary from the Nuffield Foundation. We also thank the Natural Sciences and Engineering Research Council of Canada for support. 
${ }^{1}$ L. T. Canham, Appl. Phys. Lett. 57, 1046 (1990).

${ }^{2}$ V. A. Belyakov, V. A. Burdov, R. Lockwood, and A. Meldrum, Adv. Opt. Technol 2008, 279502 (2008)

${ }^{3}$ P. F. Trwoga, A. J. Kenyon, and C. W. Pitt, Electron. Lett. 32, 1703 (1996).

${ }^{4}$ M. Sopinskyy and V. Khomchenko, Curr. Opin. Solid State Mater. Sci. 7, 97 (2003).

${ }^{5}$ G. Franzò, A. Irrera, E. C. Moreira, M. Miritello, F. Iacona, D. Sanfilippo, G. Di Stefano, P. G. Fallica, and F. Priolo, Appl. Phys. A: Mater. Sci. Process. 74, 1 (2002).

${ }^{6}$ R. J. Walters, G. I. Bourianoff, and H. A. Atwater, Nature Mater. 4, 143 (2005).

${ }^{7}$ L. Pavesi, L. Dal Negro, C. Mazzoleni, G. Franzò, and F. Priolo, Nature 408, 440 (2000).

${ }^{8}$ H. Takagi, H. Ogawa, Y. Yamazaki, A. Ishizaki, and T. Nakagiri, Appl. Phys. Lett. 56, 2379 (1990)

${ }^{9}$ A. J. Kenyon, P. F. Trwoga, C. W. Pitt, and G. Rehm, J. Appl. Phys. 79, 9291 (1996).

${ }^{10}$ K. J. Price, L. E. McNeil, A. Suvkanov, E. A. Irene, P. J. MacFarlane, and M. E. Zvanut, J. Appl. Phys. 86, 2628 (1999).

${ }^{11}$ J. S. Biteen, N. S. Lewis, H. A. Atwater, and A. Polman, Appl. Phys. Lett. 84, 5389 (2004).

${ }^{12}$ F. Koch, V. Petrova-Koch, and T. Muschik, J. Lumin. 57, 271 (1993).

${ }^{13}$ A. Meldrum, A. Hryciw, A. N. MacDonald, C. Blois, K. Marsh, J. Wang, and Q. Li, J. Vac. Sci. Technol. A 24, 713 (2006).

${ }^{14}$ S. M. Prokes, Appl. Phys. Lett. 62, 3244 (1993).

${ }^{15}$ M. Zhu, Y. Han, R. B. Wehrspohn, C. Godet, R. Etemadi, and D. Ballutaud, J. Appl. Phys. 83, 5386 (1998).
${ }^{16}$ T. Shimizu-Iwayama, S. Nakao, and K. Saitoh, Appl. Phys. Lett. 65, 1814 (1994).

${ }^{17}$ J. Derr, K. Dunn, D. Riabinina, F. Martin, M. Chaker, and F. Rosei, Physica E (Amsterdam) 41, 668 (2009).

${ }^{18}$ M. V. Wolkin, J. Jorne, P. M. Fauchet, G. Allan, and C. Delerue, Phys. Rev. Lett. 82, 197 (1999).

${ }^{19}$ D. Kovalev, J. Diener, H. Heckler, G. Polisski, N. Künzner, and F. Koch, Phys. Rev. B 61, 4485 (2000).

${ }^{20}$ J. Valenta, A. Fucikova, F. Vácha, F. Adamac, J. Humpolícková, M. Hof, I. Pelant, K. Kusová, K. Dohnalová, and J. Linnros, Adv. Funct. Mater. 18, 2666 (2008).

${ }^{21}$ Y. Kanemitsu, S. Okamoto, M. Otobe, and S. Oda, Phys. Rev. B 55, R7375 (1997).

${ }^{22}$ O. Guillois, N. Herlin-Boime, C. Reynaud, G. Ledoux, and F. Huisken, J. Appl. Phys. 95, 3677 (2004).

${ }^{23}$ M. Dovrat, Y. Goshen, J. Jedrzejewski, I. Balberg, and A. Sa'ar, Phys. Rev. B 69, 155311 (2004).

${ }^{24}$ R. Chen, J. Lumin. 102, 510 (2003).

${ }^{25}$ M. N. Berberan-Santos, E. N. Budonov, and B. Valeur, Chem. Phys. 315, 171 (2005).

${ }^{26}$ C. R. Mokry, P. J. Simpson, and A. P. Knights, J. Appl. Phys. 105, 114301 (2009).

${ }^{27}$ C. Delerue, G. Allan, C. Reynaud, O. Guillois, G. Ledoux, and F. Huisken, Phys. Rev. B 73, 235318 (2006)

${ }^{28}$ A. J. Kenyon, M. Wojdak, I. Ahmad, W. H. Loh, and C. J. Oton, Phys. Rev. B 77, 035318 (2008).

${ }^{29}$ G. Garcia, B. Garrido, P. Pellegrino, R. Ferre, J. A. Moreno, J. R. Morante, L. Pavesa, and M. Cazzanelli, Appl. Phys. Lett. 82, 1595 (2003).

${ }^{30}$ G. Allan and C. Delerue, Phys. Rev. B 75, 195311 (2007). 